Eric B. Schneider, $\mathrm{PhD}$

Sandeepa Sur, MSc, MHS

Vanessa Raymont,

$\mathrm{MBChB}, \mathrm{MSc}$,

MRCPsych

Josh Duckworth, MD

Robert G. Kowalski,

MBBCh, MS

David T. Efron, MD

Xuan Hui, MD, ScM

Shalini Selvarajah, MD, $\mathrm{MPH}$

Hali L. Hambridge, ScM

Robert D. Stevens, MD

Correspondence to

Dr. Schneider:

eschnei1@jhmi.edu

\title{
Functional recovery after moderate/severe traumatic brain injury
}

\author{
A role for cognitive reserve?
}

\section{ABSTRACT}

Objective: To evaluate the hypothesis that educational attainment, a marker of cognitive reserve, is a predictor of disability-free recovery (DFR) after moderate to severe traumatic brain injury (TBI).

Methods: Retrospective study of the TBI Model Systems Database, a prospective multicenter cohort funded by the National Institute on Disability and Rehabilitation Research. Patients were included if they were admitted for rehabilitation after moderate to severe TBI, were aged 23 years or older, and had at least 1 year of follow-up. The main outcome measure was DFR 1 year postinjury, defined as a Disability Rating Scale score of zero.

Results: Of 769 patients included, 214 (27.8\%) achieved DFR at 1 year. In total, 185 patients (24.1\%) had $<12$ years of education, while 390 (50.7\%) and 194 patients (25.2\%) had 12 to 15 years and $\geq 16$ years of education, respectively. DFR was achieved by 18 patients (9.7\%) with $<12$ years, 120 (30.8\%) with 12 to 15 years, and 76 (39.2\%) with $\geq 16$ years of education $(p<0.001)$. In a logistic regression model controlling for age, sex, and injury- and rehabilitationspecific factors, duration of education of $\geq 12$ years was independently associated with DFR (odds ratio $4.74,95 \%$ confidence interval $2.70-8.32$ for $12-15$ years; odds ratio $7.24,95 \%$ confidence interval 3.96-13.23 for $\geq 16$ years).

Conclusion: Educational attainment was a robust independent predictor of 1-year DFR even when adjusting for other prognostic factors. A dose-response relationship was noted, with longer educational exposure associated with increased odds of DFR. This suggests that cognitive reserve could be a factor driving neural adaptation during recovery from TBI. Neurology ${ }^{\circledR}$ 2014;82:1636-1642

\section{GLOSSARY}

$\mathbf{C I}=$ confidence interval; DFR = disability-free recovery; DRS = Disability Rating Scale; ED = emergency department; $\mathbf{G C S}=$ Glasgow Coma Scale; $\mathbf{O R}=$ odds ratio; TBI = traumatic brain injury

After moderate to severe traumatic brain injury (TBI), some patients experience lifelong disability while others with similar clinical characteristics achieve full recovery. This heterogeneity in outcome has been linked to patient- and injury-specific factors as well as physiologic disturbances encountered in the acute setting. However, existing prognostic models fail to provide a full or accurate account of the variance seen in TBI recovery. ${ }^{1,2}$

Cognitive reserve has been identified as a driver of the degree to which brain disorders are phenotypically expressed. ${ }^{3}$ Among patients with Alzheimer disease, the extent of pathologic damage to the brain does not correlate directly with clinical signs of dementia. ${ }^{4}$ The cognitive reserve hypothesis explains this discrepancy by proposing that individuals have inherent differences in their vulnerability to the effects of aging or brain lesions, and perhaps also in their capacity to adapt or compensate for such processes. ${ }^{3}$ The cognitive reserve hypothesis is supported by studies demonstrating intrinsic lifestyle, neuroanatomical, and functional brain

From the Center for Surgical Trials and Outcomes Research, Department of Surgery (E.B.S., D.T.E., X.H., S. Selvarajah, H.L.H.), Division of Neurosciences Critical Care, Departments of Anesthesiology and Critical Care Medicine (R.G.K., R.D.S.), and Departments of Neurology (R.D.S.) and Neurosurgery (R.D.S.), Johns Hopkins School of Medicine, Baltimore, MD; Tulane Center for Aging (S. Sur), Tulane University School of Medicine, New Orleans, LA; Department of Radiology (V.R., J.D., R.D.S.), Johns Hopkins University, Baltimore, MD; and Centre for Mental Health (V.R.), Department of Medicine, Imperial College London, UK.

Go to Neurology.org for full disclosures. Funding information and disclosures deemed relevant by the authors, if any, are provided at the end of the article. 
characteristics linked to disease expression., One of the factors most robustly associated with cognitive reserve is the level of educational attainment. Hence, educational attainment is widely accepted as a valid surrogate marker for underlying cognitive reserve. ${ }^{7}$

Studies of cognitive reserve have focused primarily on patients with evolving chronic neurodegenerative conditions such as Alzheimer disease, HIV, or multiple sclerosis., ${ }^{5,9}$ The relevance of this paradigm to the clinical expression of TBI remains poorly understood. ${ }^{10,11} \mathrm{We}$ hypothesized that educational attainment, a marker of cognitive reserve, would predict functional recovery in individuals with moderate to severe TBI.

METHODS Standard protocol approvals, registrations, and patient consents. After approval from the Institutional Review Board of the Johns Hopkins Medical Institutions and from the TBI Model Systems Study Group, we successfully applied for access to datasets collected in the TBI Model Systems cohort. The TBI Model Systems is a prospective, multicenter, observational study, supported by the National Institute on Disability and Rehabilitation Research and the US Department of Education, which examines recovery and outcomes associated with acute neurotrauma and inpatient rehabilitation. ${ }^{12}$ To be enrolled in the TBI Model Systems cohort, patients must be at least 16 years of age at the time of injury, admitted to a TBI Model Systems participating emergency department (ED) within

Figure 1 Study flow diagram

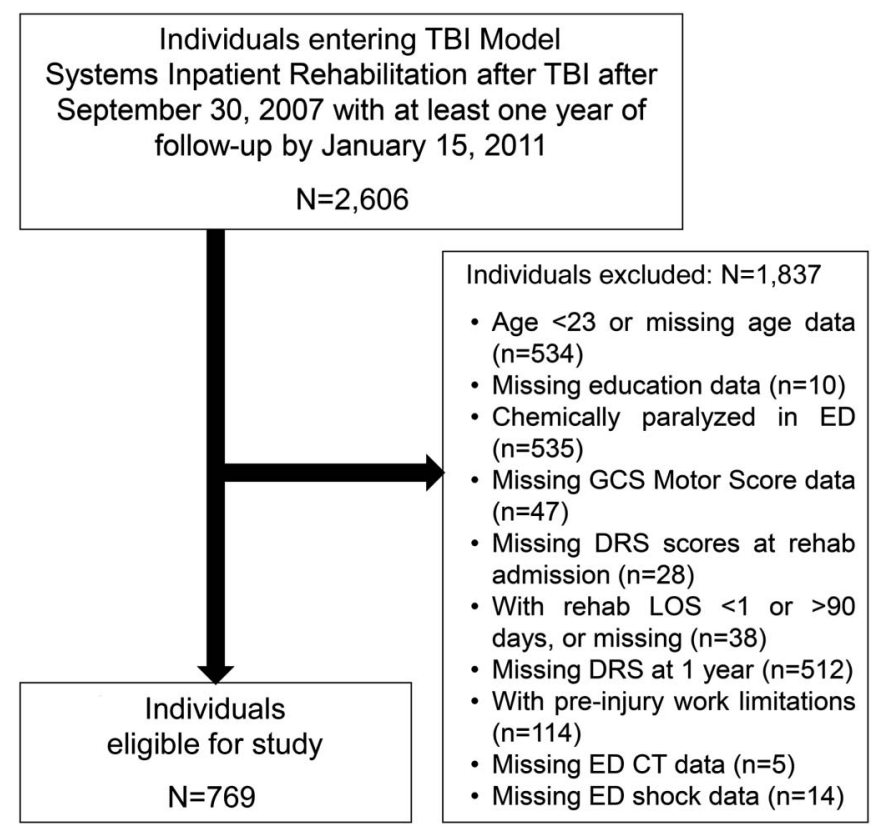

TBI Model Systems patients eligible, included, and excluded in the study, including reasons for exclusion. DRS = Disability Rating Scale; ED = emergency department; GCS = Glasgow Coma Scale; LOS = length of stay; TBI = traumatic brain injury.
24 hours of injury, receive both acute care and inpatient rehabilitation in hospitals designated as TBI Model Systems centers, and provide informed consent. Follow-up interviews of TBI Model Systems patients are conducted 12 months postinjury via a standardized assessment protocol. ${ }^{13}$

Patients were included in the current study if they had moderate to severe TBI (Glasgow Coma Scale [GCS] score on arrival in the $\mathrm{ED}$ of 12 or less), were 23 years or older at the time of injury, entered inpatient rehabilitation after September 30, 2007, and had completed at least 1 year of postinjury follow-up as of January 15, 2011. The lower age cutoff was selected so that it would be theoretically possible for all individuals to have had the opportunity to pursue formal education for 16 years at the time of their injury. Subjects were excluded from the study if their education level and other important information concerning their demographics, injury, or outcome were not available. Individuals were also excluded if there was an indication of any premorbid physical or mental condition that limited their ability to work (see figure 1).

The primary predictor variable of interest was educational attainment measured in years. Specifically, education was examined as a categorical variable with the first category corresponding to an incomplete high school education ( $<12$ years of education), the second corresponding to high school completion with some postsecondary education (12-15 years of education), and the third corresponding to sufficient years of education to achieve at least an undergraduate degree ( $\geq 16$ years of education).

The primary outcome variable was disability-free recovery (DFR), defined as a score of zero on the Disability Rating Scale (DRS) assessed 1 year postinjury. The DRS is a quantitative instrument that has been extensively validated in the TBI population. ${ }^{1,14}$ Components of the DRS include assessments of arousal and awareness, ability to handle self-care functions, physical dependence on others, and psychosocial adaptability for work, housework, or school. The scores range from zero (no disability) to 29 (complete disability). The DRS score was dichotomized between zero and greater than zero because this demarcation is thought to be highly relevant from a patient-centered standpoint. A DRS of zero is defined as the ability to completely resume preinjury occupational activity (work or education). While a DRS score of zero indicates freedom from significant disability, this scale is not designed to evaluate cognitive impairments, and a DRS score of zero does not exclude the possibility of subtle functional challenges.

Statistical analysis. We identified subjects who achieved DFR and compared their characteristics with those who did not (DRS score $>0$ at 1 year postinjury). Univariate differences in group variables were examined using parametric and nonparametric tests where appropriate (Student $t$ test, Wilcoxon rank-sum test, analysis of variance, and $\chi^{2}$ test). Multivariable logistic regression was used to quantify the relationship between educational attainment and DFR, adjusting for factors known or observed to be associated with educational attainment or functional outcome. The final logistic regression models examined the odds of achieving a DFR (DRS score $=0$ ) across education levels, controlling for age, sex, GCS motor score at the time of arrival in the ED, the presence of circulatory shock in the ED, the presence of subarachnoid hemorrhage or intraventricular hemorrhage, or brain compression on initial CT scan, as well as DRS score at admission to rehabilitation. An age-based subset logistic regression analysis was conducted examining the relationship between educational attainment and DFR in individuals aged 50 years and older using the same modeling parameters above. Also, while the principal outcome of interest was DFR, analysis 
Table 1 Patient demographics and injury characteristics by level of education $(N=769)$

\begin{tabular}{|c|c|c|c|c|}
\hline & $\begin{array}{l}<12 \text { y of education } \\
(n=185)\end{array}$ & $\begin{array}{l}12-15 y \text { of education } \\
(n=390)\end{array}$ & $\begin{array}{l}\geq 16 \text { y of education } \\
(n=194)\end{array}$ & $p$ Value \\
\hline Mean age, y (SD) & 46.9 (16.8) & $48.6(17.8)$ & $54.3(17.7)$ & $<0.001$ \\
\hline Female, $\mathrm{n}(\%)$ & 33 (17.8) & 118 (30.3) & $57(29.4)$ & 0.006 \\
\hline Race, $n(\%)$ & & & & $<0.001$ \\
\hline White & 96 (51.9) & $281(72.1)$ & $161(83.0)$ & \\
\hline Black & $52(28.1)$ & 74 (19.0) & $11(5.7)$ & \\
\hline Hispanic & 34 (18.4) & $24(6.2)$ & $3(1.6)$ & \\
\hline Asian & $2(1.1)$ & $7(1.8)$ & $16(8.3)$ & \\
\hline Other & $1(0.5)$ & $4(1.0)$ & $3(1.6)$ & \\
\hline Mean ED GCS motor score (SD) & 4.5 (1.9) & $4.6(1.8)$ & 4.9 (1.8) & 0.094 \\
\hline SAH, n (\%) & 133 (71.9) & $273(70.0)$ & $133(68.6)$ & 0.777 \\
\hline IVH, n (\%) & 44 (23.8) & $109(26.0)$ & 46 (23.7) & 0.413 \\
\hline Compression, n (\%) & 94 (49.2) & 149 (38.2) & $82(42.3)$ & 0.044 \\
\hline Circulatory shock, n (\%) & $5(2.7)$ & $15(3.9)$ & $3(1.6)$ & 0.297 \\
\hline Mean DRS score at admission (SD) & $11.1(4.9)$ & $11.0(4.9)$ & 10.7 (5.2) & 0.688 \\
\hline Rehabilitation length of stay, $d(S D)$ & 23.9 (16.5) & $23.3(16.1)$ & 22.9 (13.7) & 0.815 \\
\hline
\end{tabular}

Abbreviations: DRS = Disability Rating Scale; ED = emergency department; GCS = Glasgow Coma Scale; IVH = intraventricular hemorrhage; SAH = subarachnoid hemorrhage.

of variance and Kruskal-Wallis tests were used to examine DRS scores by education group, both at the time of admission to rehabilitation and 1 year postinjury.

To further explore the possibility of dose response, a regression model was run whereby education was grouped into 4 categories as follows: incomplete high school education $(<12$ years of education), high school completion with or without some postsecondary education (12-15 years of education), sufficient years of education to achieve an undergraduate degree with or without some postgraduate education (16-20 years of education), and 4

Figure 2 DRS scores at admission to rehabilitation and at 1 year

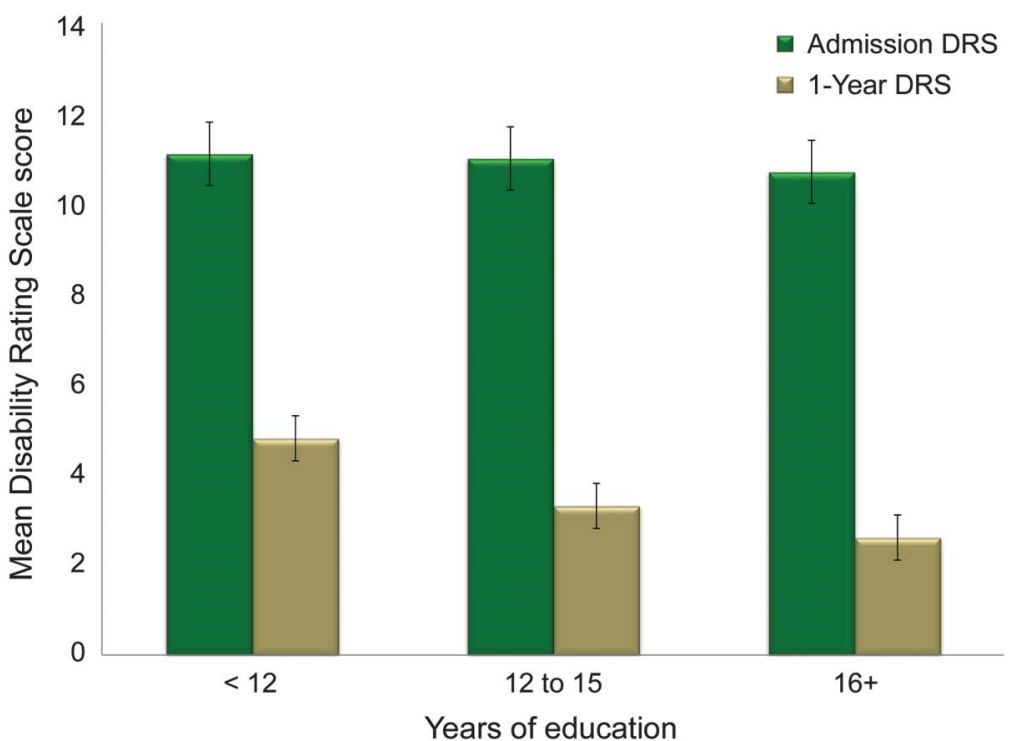

Mean (95\% confidence interval) Disability Rating Scale (DRS) scores on admission to rehabilitation and at 1 year in patients grouped by years of education. or more years of education beyond the undergraduate degree ( $\geq 20$ years of education).

RESULTS From 2,606 records examined, a total of 769 patients met inclusion criteria and underwent analysis (figure 1). Among these, 214 (27.8\%) achieved DFR at 1 year. Overall, 185 subjects (24.1\%) had fewer than 12 years of education, 390 $(50.7 \%)$ had 12 to 15 years of education, and 194 (25.2\%) had 16 or more years of education. The youngest individual in the study sample to have completed 16 years of education was 23 years of age.

Characteristics of the study population are detailed in table 1 . The mean age of patients with 16 or more years of education was 54.3 years, compared with 48.6 years in the group with 12 to 15 years of education and 46.9 years in the group with fewer than 12 years of education $(p<0.001)$. Subjects with fewer than 12 years of education were less likely to be female $(17.8 \%)$, compared with those who had 12 to 15 years $(30.3 \%)$ and those with 16 or more years (29.4\%) of education $(p=0.006)$. Individuals with at least 16 years of education were more likely to be white $(83.0 \%)$, compared with those who had 12 to 15 years $(72.1 \%)$ or those with fewer than 12 years $(51.9 \%)$ of education $(p<0.001)$. Mean GCS motor score in the ED was higher in the group with 16 or more years of education (4.9), compared with those who had 12 to 15 years (4.6) and those with fewer than 12 years (4.5) of education, but this difference was not significant $(p<0.094)$. Motor vehicle crashes were the most common mechanism of injury 
across all groups, involving $47.0 \%$ of those subjects with fewer than 12 years of education, $53.6 \%$ of those with 12 to 15 years, and $44.9 \%$ of those with 16 or more years; falls were less common in individuals with fewer than 12 years $(30.8 \%)$ or 12 to 15 years $(30.3 \%)$ compared with those having 16 or more years $(42.3 \%)$ of education, with other mechanisms of injury being most common among those with fewer than $12(22.2 \%)$ vs 12 to $15(15.9 \%)$ or 16 or more $(12.9 \%)$ years of education $(p=0.024)$. No other significant differences were observed across the 3 education groups for either demographic or injuryrelated variables. There was also no difference across education groups for mean DRS score at admission to inpatient rehabilitation or length of stay in inpatient rehabilitation.

One year after injury, DFR was achieved by 214 patients $(27.8 \%)$, of whom $18(9.7 \%)$ had fewer than 12 years of education, $120(30.8 \%)$ had 12 to 15 years, and $76(39.2 \%)$ had 16 or more years of education $(p<$ 0.001). Overall, mean DRS scores at the time of admission did not differ across education groups (11.1
[SD 4.9] for $<12$ years vs 11.0 [SD 4.9] for 12-15 years vs 10.7 [SD 5.2] for $\geq 16$ years; $p=0.688$ ); however, mean DRS scores were significantly different across groups at 1 year postinjury (3.9 [SD 3.5] for $<12$ years vs 2.4 [SD 2.9] for $12-15$ years vs 2.0 [SD 3.1] for $\geq 16$ years; $p<0.001$ ) (figure 2 ). The distributions of DRS scores at the time of admission rehabilitation and at 1 year postinjury are represented in figure 3 .

In multivariable logistic regression, educational attainment was independently associated with DFR (odds ratio [OR] 4.74, 95\% confidence interval [CI] 2.70-8.32 for $12-15$ years; OR 7.24, 95\% CI $3.96-13.23$ for $\geq 16$ years). Increased patient age and rehabilitation length of stay were associated with reduced odds of DFR (OR 0.41, 95\% CI 0.280.62 and OR $0.97,95 \%$ CI 0.96-0.99, respectively), and higher DRS scores at admission, by quartile, demonstrated (quartiles 2 and 4) or trended toward (quartile 3) reduced odds of DFR (see table 2).

In a sensitivity analysis of subjects aged 50 years and older $(\mathrm{n}=341)$, education retained its independent association with DFR (OR 4.11, 95\% CI

Figure 3 DRS score distribution
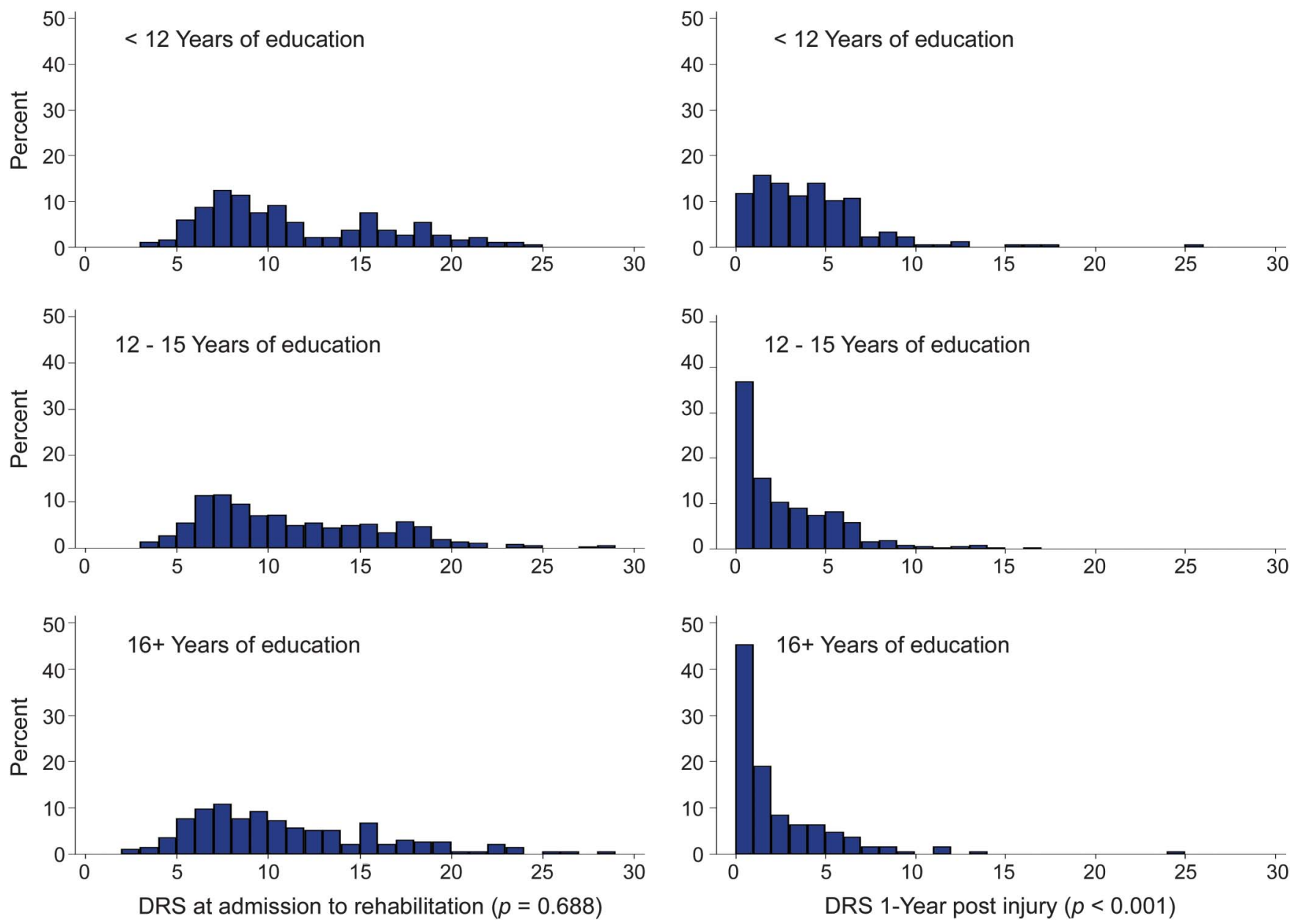

Distribution of Disability Rating Scale (DRS) scores on admission to rehabilitation and 1 year postinjury in patients grouped by years of education. 


\section{Table 2 Multivariate odds of DFR}

DFR (DRS score $=0$ )

OR

$95 \% \mathrm{Cl}$

Education and demographics

\begin{tabular}{|c|c|c|}
\hline $12-15$ y of education & 4.74 & $2.70-8.32$ \\
\hline$\geq 16$ y of education & 7.24 & $3.96-13.23$ \\
\hline Age $>40 y$ & 0.41 & $0.28-0.62$ \\
\hline Female & 1.24 & $0.83-1.85$ \\
\hline \multicolumn{3}{|l|}{ njury/ED factors } \\
\hline Shock & 0.22 & $0.06-1.16$ \\
\hline Revised Trauma Scale (per point) & 1.00 & $1.00-1.00$ \\
\hline \multicolumn{3}{|l|}{ ED GCS motor score } \\
\hline 1 & 0.82 & $0.43-1.56$ \\
\hline 2 & 0.18 & $0.02-1.47$ \\
\hline 3 & 0.28 & $0.06-1.28$ \\
\hline 4 & 0.64 & $0.34-1.21$ \\
\hline 5 & 1.40 & $0.85-2.30$ \\
\hline \multicolumn{3}{|l|}{ TT findings } \\
\hline Subarachnoid hemorrhage & 1.41 & $0.95-2.10$ \\
\hline Intraventricular hemorrhage & 0.71 & $0.46-1.10$ \\
\hline Compression & 0.80 & $0.56-1.15$ \\
\hline \multicolumn{3}{|l|}{ Rehabilitation factors } \\
\hline Admission DRS quartile 2 & 0.60 & $0.38-0.95$ \\
\hline Admission DRS quartile 3 & 0.63 & $0.39-1.01$ \\
\hline Admission DRS quartile 4 & 0.47 & $0.25-0.88$ \\
\hline Rehabilitation length of stay (per additional day) & 0.97 & $0.96-0.99$ \\
\hline
\end{tabular}

Abbreviations: $\mathrm{Cl}=$ confidence interval; DFR = disability-free recovery; DRS = Disability Rating Scale; ED = emergency department; GCS = Glasgow Coma Scale; OR = odds ratio. Reference groups: $<12$ years of education, male, ED GCS score $=6$, highest functioning (first quartile of DRS) at rehabilitation admission.

$1.71-9.88$ for $12-15$ years; OR 5.22 , 95\% CI 2.12 12.83 for $\geq 16$ years); aside from educational attainment, the only other significant predictor of DFR was high disability on admission to rehabilitation (lowest DRS score quartile, OR 0.32, 95\% CI 0.12-0.85).

A further multivariate analysis was completed to evaluate individuals with 20 or more years of education $(\mathrm{n}=32)$. In this model, individuals with 20 or more years of education had a 9-fold increased odds of DFR compared with individuals with fewer than 12 years of education (OR 9.33, 95\% CI 3.7823.01), adjusting for the factors mentioned above.

DISCUSSION Our findings, obtained from a large, prospective, multicenter cohort, demonstrate that more than one-quarter of patients with moderate or severe TBI who undergo intensive rehabilitation are free of significant functional impairment 1 year after injury. In addition, we found that preinjury educational attainment is a strong independent predictor of longterm functional outcome in this population. The plausibility of this result is supported by a graded "dose-response" relationship whereby higher levels of educational attainment were associated with greater odds of achieving functional independence. The association between education and functional outcome was maintained even after adjustment for other major outcome classifiers. Taken together, these data indicate that educational status, a marker of cognitive reserve, is a major and largely unaccounted determinant of functional recovery after TBI.

A substantial body of evidence generated from the study of patients with progressive neurologic disorders, such as Alzheimer disease, HIV encephalopathy, and multiple sclerosis, indicates that cognitive reserve is a fundamental factor modulating disease expression. ${ }^{8,15,16}$ Despite these converging results, a unifying biological theory of cognitive reserve is lacking, highlighting the need for hypothesis-driven translational research in this area. ${ }^{3}$ Recent studies suggest that the variability in recovery from TBI may be driven by differences in brain anatomical ${ }^{17}$ and functional ${ }^{18,19}$ connectivity. While such connectivity differences may reflect heterogeneity in the severity of injury, it is also possible that they reflect preinjury differences in cognitive reserve. Research is needed to unravel the complex relationships among preinjury variables, including cognitive reserve, injury severity, and postinjury reparative and compensatory factors. Clarification of cognitive reserve biology and its link to neural plasticity could identify targets for interventions to improve outcomes for patients with neurologic disease and injury, including TBI. ${ }^{20}$

A few clinical studies support the view that cognitive reserve modulates recovery after acute disorders such as ischemic stroke ${ }^{21}$ and TBI. ${ }^{10,11}$ In a study of 25 patients who underwent brain MRI scanning before TBI, patients with lower preinjury total intracranial volume and educational status had lower postinjury IQ regardless of injury severity. ${ }^{10}$ In a retrospective analysis of 52 children who had neuropsychological testing before and after TBI, premorbid test scores were not predictive of postinjury performance. ${ }^{11}$ Neither of these 2 studies evaluated functional outcome, nor was there any adjustment for covariates that might contribute to post-TBI outcome.

Our data indicate a robust association between educational attainment and functional recovery in a large sample of patients after TBI; however, limitations of the study must be recognized. First, it is important to acknowledge that educational attainment is a surrogate, and not a direct, marker of cognitive reserve. While available published research supports the construct of education as a marker of reserve, it remains unclear whether higher educational achievement is causatively linked to greater cognitive reserve, results from it, or both. ${ }^{7,16}$ Educational attainment itself is not solely reflective of intellectual or cognitive abilities. Motivation to succeed and self-discipline, as well as 
socioeconomic status, are likely also associated with higher levels of education and may have important roles in determining the degree of post-TBI recovery. A second limitation is that only patients admitted to TBI Model Systems inpatient rehabilitation facilities were studied; therefore, findings may not be generalizable to the other TBI populations. Third, only patients with moderate to severe TBI were included, and although we suspect that cognitive reserve may have an important role in functional outcomes among individuals with milder forms of TBI, this cannot be extrapolated from our data. Fourth, beyond whether preinjury work limitations existed, we did not evaluate the role of preinjury occupational or socioeconomic status (the data were not available), other physical or

\section{Comment:}

\section{Importance of cognitive reserve in traumatic brain injury}

The expectation for moderate to severe traumatic brain injury (TBI) is permanent damage and lasting deficits. However, in a multicenter investigation, Schneider et al. ${ }^{1}$ show that by 1 year postinjury, one-fourth of patients with TBI achieve disability-free recovery (DFR), defined as a score of zero on the Disability Rating Scale. Of importance, cognitive reserve (CR) in the form of educational attainment was related to DFR.

The neural basis for learning and memory depends on network integrity. Functional neuroimaging studies show the modifiability and strengthening of large-scale networks by educational training. ${ }^{2}$ In this sense, educational attainment likely represents a proxy for neural processes that reflect network integrity.

Once injured, how networks mend or adapt determines TBI outcome. Does educational attainment function passively, whereby established networks provide the neural scaffolding for rerouting and adaption? Or is there something more active that leads to DFR? If educational attainment implicates more efficient neural networks, established networks may be better able to respond to the treatment milieu associated with intervention and rehabilitation after TBI. Regardless, these findings provide intriguing hypotheses about the role of CR in brain structure and function as well as response to injury.

Sumowski et al. ${ }^{3}$ have shown that educational attainment also attenuates the level of cognitive impairment in TBI. CR is a major factor related to resiliency in aging and the timing and expression of dementing illnesses. The investigations by Schneider et al. ${ }^{1}$ and others ${ }^{3,4}$ firmly establish a CR role in TBI outcome.

Of course, caution is needed so as not to overinterpret the role of educational attainment. The Disability Rating Scale is but a rating scale with low and overly broad thresholds that lead to DFR classification. Nonetheless, because the brain is an experience-dependent organ, educational attainment must reflect neural integrity at some level, which in turn represents an important factor for the clinician to consider in understanding TBI outcome.

1. Schneider EB, Sur S, Raymont V, et al. Functional recovery after moderate/severe traumatic brain injury: a role for cognitive reserve? Neurology 2014;82:1636-1642.

2. Mackey AP, Miller Singley AT, Bunge SA. Intensive reasoning training alters patterns of brain connectivity at rest. J Neurosci 2013;33:4796-4803.

3. Sumowski JF, Chiaravalloti N, Krch D, Paxton J, Deluca J. Education attenuates the negative impact of traumatic brain injury on cognitive status. Arch Phys Med Rehabil 2013;94:2562-2564

4. Levi Y, Rassovsky Y, Agranov E, Sela-Kaufman M, Vakil E. Cognitive reserve components as expressed in traumatic brain injury. J Int Neuropsychol Soc 2013;19:664-671.

\section{Erin D. Bigler, $\mathrm{PhD}$}

From the Magnetic Resonance Imaging Research Facility, Brigham Young University, Provo, and University of Utah School of Medicine, Salt Lake City.

Study funding: No targeted funding reported.

Disclosure: E. Bigler serves on the executive advisory board for the Department of Defense, Injury and Traumatic Stress Consortium and on the scientific advisory board, HIV Neurobehavioral Research Program, University of California at San Diego; serves as an associate editor for Brain Imaging and Behavior and Neuropsychology; receives publishing rovalties from Oxford University Press; receives research support from the NIH and the National Institute of Mental Health; and has given expert testimony in a medicolegal case. Go to Neurology.org for full disclosures. psychiatric difficulties, or alcohol/substance abuse, each of which might be significant effect modifiers in TBI recovery. Nevertheless, recent work in cohorts of elderly patients indicates that educational attainment is a more robust and independent predictor of later cognitive performance than occupation. ${ }^{7}$ Fifth, a single year of follow-up may be insufficient to enable an adequate understanding of how cognitive reserve, as measured by educational attainment, relates to lifelong functional ability in patients with TBI. Sixth, in analysis of subsets of patients, particularly the subset with more than 20 years of education, the small number of eligible individuals limits our power to definitively detect meaningful differences. Seventh, it is possible that our exclusion of patients who had been chemically paralyzed in the ED or who were missing ED GCS data, or those for whom 1-year DRS scores were unavailable may have biased our results substantially. Lastly, the DRS is a relatively simple tool that is heavily biased toward the assessment of functional capacity and may fail to capture subtle but clinically important neuropsychological impairments.

Recovery without disability, as measured using the DRS, was achieved in a substantial proportion of subjects with moderate to severe TBI who underwent inpatient rehabilitation. This study provides evidence that educational attainment, a marker of cognitive reserve, is associated with a substantially increased likelihood of recovery in this setting. Research is needed to elucidate the biological mechanisms of cognitive reserve and how this process is related to learning, education, injury, and resilience. It is expected that exploring these relationships will help identify interventions to promote greater degrees of recovery after TBI.

\section{AUTHOR CONTRIBUTIONS}

Drs. Schneider and Stevens had full access to all of the data in the study and take full responsibility for the integrity of the data and the accuracy of the data analysis. Study concept and design: Drs. Schneider, Stevens, Kowalski, Efron, Duckworth, and Mss. Sur and Hambridge. Acquisition of data: Drs. Schneider and Stevens and Ms. Hambridge. Analysis and interpretation of data: Drs. Schneider, Stevens, Hui, Selvarajah, Kowalski, Duckworth, Efron, and Mss. Sur and Hambridge. Drafting of the manuscript: Drs. Schneider, Stevens, Raymont, Selvarajah, and Ms. Sur. Critical revision of the manuscript for important intellectual content: Drs. Schneider, Stevens, Raymont, Kowalski, Efron, Duckworth, Mss. Sur and Hambridge. Statistical analysis: Drs. Schneider, Selvarajah, Hui, Stevens, and Ms. Hambridge. Administrative, technical, or material support: Dr. Selvarajah and Ms. Hambridge. Study supervision: Drs. Schneider and Stevens.

\section{ACKNOWLEDGMENT}

The authors acknowledge the assistance of the TBI Model Systems National Data and Statistical Center; specifically, Dr. Cindy Harrison-Felix, Mr. Chris Cusik, and Dr. Chris Pretz for their assistance in providing study data, as well as the National Institute on Disability and Rehabilitation Research and the US Department of Education, which support data collection and maintenance within the TBI Model Systems program.

\section{STUDY FUNDING}

No external funding was provided for this study. The collection of the original data was supported by the National Institute on 
Disability and Rehabilitation Research and the U.S. Department of Education.

\section{DISCLOSURE}

E. Schneider has pending patents on a treatment for acute CNS injury, which are not related to the content of this report. S. Sur, V. Raymont, J. Duckworth, R. Kowalski, D. Efron, X. Hui, S. Selvarajah, and H. Hambridge report no disclosures relevant to the manuscript. R. Stevens has received funding from the Defense Advanced Research Projects Agency, the Department of Defense, and from the Johns Hopkins Brain Sciences Institute. Go to Neurology.org for full disclosures.

Received July 18, 2013. Accepted in final form January 21, 2014.

\section{REFERENCES}

1. Murray GD, Butcher I, McHugh GS, et al. Multivariable prognostic analysis in traumatic brain injury: results from the IMPACT study. J Neurotrauma 2007;24:329.

2. Perel P, Arango M, Clayton T, et al. Predicting outcome after traumatic brain injury: practical prognostic models based on large cohort of international patients. BMJ 2008;336:425.

3. Stern Y. Cognitive reserve in ageing and Alzheimer's disease. Lancet Neurol 2012;11:1006.

4. Katzman R, Terry R, DeTeresa R, et al. Clinical, pathological, and neurochemical changes in dementia: a subgroup with preserved mental status and numerous neocortical plaques. Ann Neurol 1988;23:138.

5. Rentz DM, Locascio JJ, Becker JA, et al. Cognition, reserve, and amyloid deposition in normal aging. Ann Neurol 2010;67:353-364.

6. Stern Y, Zarahn E, Habeck C, et al. A common neural network for cognitive reserve in verbal and object working memory in young but not old. Cereb Cortex 2008;18: 959-967.

7. Murray AD, Staff RT, McNeil CJ, et al. The balance between cognitive reserve and brain imaging biomarkers of cerebrovascular and Alzheimer's diseases. Brain 2011; 134:3687.

8. Chang L, Tomasi D, Yakupov R, et al. Adaptation of the attention network in human immunodeficiency virus brain injury. Ann Neurol 2004;56:259.

9. Sumowski JF, Wylie GR, DeLuca J, Chiaravalloti N. Intellectual enrichment is linked to cerebral efficiency in multiple sclerosis: functional magnetic resonance imaging evidence for cognitive reserve. Brain 2010;133:362-374.
10. Kesler SR, Adams HF, Blasey CM, Bigler ED. Premorbid intellectual functioning, education, and brain size in traumatic brain injury: an investigation of the cognitive reserve hypothesis. Appl Neuropsychol 2003;10:153.

11. Fuentes A, McKay C, Hay C. Cognitive reserve in paediatric traumatic brain injury: relationship with neuropsychological outcome. Brain Inj 2010;24:995.

12. Sherer M, Sander AM, Nick TG, High WM, Malec JF, Rosenthal M. Early cognitive status and productivity outcome after traumatic brain injury: findings from the TBI model systems. Arch Phys Med Rehabil 2002;83:183.

13. Brooks CA, Gabella B, Hoffman R, Sosin D, Whiteneck G. Traumatic brain injury: designing and implementing a population-based follow-up system. Arch Phys Med Rehabil 1997;78(suppl 4):S26-S30.

14. Rappaport M, Hall KM, Hopkins K, Belleza T, Cope DN. Disability Rating Scale for severe head trauma: coma to community. Arch Phys Med Rehabil 1982;63:118.

15. Cader S, Cifelli A, Abu-Omar Y, Palace J, Matthews PM. Reduced brain functional reserve and altered functional connectivity in patients with multiple sclerosis. Brain 2006;129:527.

16. Kemppainen NM, Aalto S, Karrasch M, et al. Cognitive reserve hypothesis: Pittsburgh compound B and fluorodeoxyglucose positron emission tomography in relation to education in mild Alzheimer's disease. Ann Neurol 2008;63:112.

17. Galanaud D, Perlbarg V, Gupta R, et al. Assessment of white matter injury and outcome in severe brain trauma: a prospective multicenter cohort. Anesthesiology 2012;117: 1300-1310.

18. Palacios EM, Sala-Llonch R, Junque C, et al. Resting-state functional magnetic resonance imaging activity and connectivity and cognitive outcome in traumatic brain injury. JAMA Neurol 2013;70:845-851.

19. Pandit AS, Expert P, Lambiotte, et al. Traumatic brain injury impairs small-world topology. Neurology 2013; 80:1826-1833.

20. Levi Y, Rassovsky Y, Agranov E, Sela-Kaufman M, Vakil E. Cognitive reserve components as expressed in traumatic brain injury. J Int Neuropsychol Soc 2013;19:664-671.

21. Elkins JS, Longstreth WT, Manolio TA, Newman AB, Bhadelia RA, Johnston SC. Education and the cognitive decline associated with MRI-defined brain infarct. Neurology 2006;67:435.

\section{Subspecialty Alerts by E-mail!}

Customize your online journal experience by signing up for e-mail alerts related to your subspecialty or area of interest. Access this free service by visiting http://www.neurology.org/site/subscriptions/etoc.xhtml or click on the "E-mail Alerts" link on the home page. An extensive list of subspecialties, methods, and study design choices will be available for you to choose from-allowing you priority alerts to cutting-edge research in your field! 